\title{
General Localization Lengths for Two Interacting Particles in a Disordered Chain
}

\author{
Pil Hun Song and Felix von Oppen \\ Max-Planck-Institut für Kernphysik, Postfach 103980, 69029 Heidelberg, Germany
}

(May 1, 2018)

\begin{abstract}
The propagation of an interacting particle pair in a disordered chain is characterized by a set of localization lengths which we define. The localization lengths are computed by a new decimation algorithm and provide a more comprehensive picture of the two-particle propagation. We find that the interaction delocalizes predominantly the center-of-mass motion of the pair and use our approach to propose a consistent interpretation of the discrepancies between previous numerical results.

PACS number(s): 72.15.Rn, 71.30.+h
\end{abstract}

The problem of interacting electrons in a disordered potential is one of the important unsolved problems in condensed matter physics. This has been emphasized again by the recent observation [1] of a metal-insulator transition in two dimensional $(2 \mathrm{~d})$ systems which was theoretically unanticipated. Some time ago, Shepelyansky [2] proposed that it would be worthwhile to consider the simple case of two interacting particles in a random potential. He predicted that unexpectedly, such a particle pair could propagate coherently over distances $\xi_{2}$ much larger than the single-particle localization length $\xi_{1}$ as long as the two particles are within $\xi_{1}$ from each other.

Specifically, Shepelyansky obtained for the twoparticle localization length

$$
\xi_{2} \sim\left(U / W^{2}\right)^{2},
$$

where $U$ denotes the interaction strength and $W$ the disorder strength. Since $\xi_{1} \sim 1 / W^{2}$, Eq. (1) implies an enhancement of the localization length for weak disorder. Shepelyansky's original argument involved several uncontrolled assumptions for the single-particle eigenstates. This led to a number of (mostly numerical) attempts [3] to study the problem of two interacting particles more rigorously. Imry [3] rederived Shepelyansky's result, Eq. (1), by an extension of the Thouless block

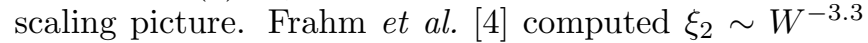
using the transfer matrix method (TMM). Von Oppen et al. [5] introduced a Green function approach, allowing one to project the problem on the subspace of doubly occupied sites, and concluded $\xi_{2} \sim U / W^{4}$. Subsequently, Song and Kim [6] treated the idea of von Oppen et al. rigorously using the recursive Green function method and found $\xi_{2} \sim W^{-2.9}$.

Recently, Römer and Schreiber [7] concluded from the TMM that the enhancement effect does not exist. In view of this claim and of the quantitatively different expressions for $\xi_{2}$ quoted above, it appears that there are few secured results in this field. Our purpose in this paper is to present a more comprehensive picture of the twoparticle propagation by defining and computing a set of localization lengths. We unambiguously show that the effect exists and propose a resolution of the controversy in the previous works [7,10].
It is currently not clear whether these ideas have any relevance to the degenerate finite-density Fermi gas. It appears to be the most promising direction to consider the localization properties of quasiparticle pairs. There have been a number of studies [3,8.,9] whether quasiparticle excitations delocalize relative to single-particle ones. While a numerical study for a one-dimensional (1d) system showed delocalization only for unrealistically high excitation energy of the pair (of order of the bandwidth) [8], both arguments [3] and numerical studies [9] in higher dimensions suggest the possibility of a new pair mobility edge close to the ground state.

The two-particle problem in one-dimension is described by the Hamiltonian

$$
\mathcal{H}=H_{1} \otimes \mathbf{1}+\mathbf{1} \otimes H_{1}+U \sum_{m}|m\rangle|m\rangle\langle m|\langle m|,
$$

where $m$ labels the $N$ sites of the 1 d lattice and $H_{1}$ is the usual single-particle Anderson Hamiltonian

$$
H_{1}=\sum_{m}\left[\epsilon_{m}|m\rangle\langle m|+t(|m\rangle\langle m+1|+| m+1\rangle\langle m|)\right] .
$$

$\epsilon_{m}$ is a random site energy, drawn from a box distribution with $-W / 2 \leq \epsilon_{m} \leq W / 2$, and $U$ the on-site interaction. The hopping matrix element $t$ is set to unity throughout this work. A convenient quantity to study the localization properties of the pair is the two-particle Green function $G=(E-\mathcal{H})^{-1}$. The two-particle localization length $\xi_{2}$ on which previous studies have focused is defined in terms of $G$ as [5]

$$
\xi_{2}^{-1}=-\lim _{|n-m| \rightarrow \infty} \frac{1}{|n-m|} \ll \ln |\langle m, m|G| n, n\rangle| \gg,
$$

where the double bracket denotes the disorder average.

In this paper, we discuss general localization lengths which provide a much more comprehensive picture of the localization properties of the particle pair. First, we consider a general center-of-mass (CM) motion by defining

$$
\xi_{2, a}^{-1}=-\lim _{|n-m| \rightarrow \infty} \frac{1}{|n-m|} \ll \ln |\langle m, m-a|G| n, n-a\rangle| \gg .
$$


We find that, surprisingly, $\xi_{2, a}$ is essentially independent of the particle distance $a$, even if $a$ exceeds the singleparticle localization length $\xi_{1}$. We also study the behavior of $G$ for relative motion at fixed $\mathrm{CM}$, as characterized by

$$
\xi_{r}^{-1}=-\lim _{n \rightarrow \infty} \frac{1}{n} \ll \ln |\langle m+n, m-n|G| m, m\rangle| \gg .
$$

Finally, we consider the propagation of one of the particles with the other held fixed, as described by

$$
\xi_{f}^{-1}=-\lim _{n \rightarrow \infty} \frac{1}{n} \ll \ln |\langle m, m+n|G| m, m\rangle| \gg .
$$

As opposed to $\xi_{2}$ and $\xi_{2, a}$, we find that the latter two lengths are only very weakly affected by the interaction $U$. Nevertheless, it will turn out that these lengths are indispensable for obtaining a more comprehensive picture of the two-particle propagation and for understanding the discrepancies between previous numerical results.

While $\xi_{2}$ could be computed by projecting the problem on the subspace of doubly occupied sites, this is no longer possible for the generalized localization lengths defined above. For this reason, we introduce a new decimation algorithm, which allows us to compute these localization lengths efficiently. As opposed to the projection method for $\xi_{2}$ used in ref. [5], this algorithm is numerically exact. We briefly describe the procedure for computing $\xi_{2}$. Adaption to the other lengths defined above is straightforward. Since the interaction acts only on symmetric states, we specify to (spinless) bosons. Using a symmetrized basis

$$
|m n\rangle= \begin{cases}|m\rangle|m\rangle & \text { if } m=n, \\ (1 / \sqrt{2})(|m\rangle|n\rangle+|n\rangle|m\rangle) & \text { if } m \neq n .\end{cases}
$$

and interpreting $(m, n)$ as sites of a $2 \mathrm{~d}$ square lattice, the Hamiltonian of Eq. (2) can be interpreted as describing a single particle on the $2 \mathrm{~d}$ lattice shown by the thin solid lines in Fig. 1. The off-diagonal elements of $\mathcal{H}$ are nonzero only for nearest-neighbor bonds and equal to $\sqrt{2}$ (1) if one (none) of the nearest-neighbor sites is a doubly occupied state. Our goal is to compute the Green function $G(E)$ which is the inverse of a sparse matrix, $D=E-\mathcal{H}$ of linear size $\sim N^{2}$. Clearly, a direct manipulation of the whole matrix is inefficient both in terms of time and storage, and becomes forbidding for $N>100$. To circumvent this problem, we recursively decimate the irrelevant matrix elements of the Green function. We start by decomposing Hilbert space into subspaces $i$, each of which is spanned by the states along one of the dashed lines in Fig. 1(a) and which are labeled by their dimensions $1 \leq i \leq N$. We denote the projection of $D$ onto these subspaces as $D_{i}$. Clearly, $D$ couples only neighboring subspaces $(i)$ and $(i+1)$, and we call the corresponding $(i \times(i+1)$ dimensional $)$ coupling block in the Hamiltonian $V_{i}$. Finally, we define vectors $\mathbf{x}_{i}^{(n)}$ with elements

$$
\left(\mathbf{x}_{i}^{(n)}\right)_{j}=\langle N-i+j, j|G(E)| n n\rangle,
$$

given by matrix elements of the Green function $G$ between a doubly occupied site $|n n\rangle$ and the states in subspace $i$. Since only neighboring subspaces are coupled, one readily derives from $D G=1$ the set of coupled linear equations

$$
\begin{aligned}
D_{1} \mathbf{x}_{1}^{(n)}+V_{1} \mathbf{x}_{2}^{(n)} & =0, \\
V_{i-1}^{T} \mathbf{x}_{i-1}^{(n)}+D_{i} \mathbf{x}_{i}^{(n)}+V_{i} \mathbf{x}_{i+1}^{(n)} & =0, \quad 2 \leq i \leq N-1, \\
V_{N-1}^{T} \mathbf{x}_{N-1}^{(n)}+D_{N} \mathbf{x}_{N}^{(n)} & =\mathbf{e}_{n},
\end{aligned}
$$

where $\mathbf{e}_{n}$ is the $N$ dimensional unit vector with $\left(\mathbf{e}_{n}\right)_{m}=$ $\delta_{n, m}$. Solving these equations, we obtain

$$
\mathbf{x}_{N}^{(n)}=\mathcal{G}_{N} \mathbf{e}_{n},
$$

where the $\mathcal{G}_{i}$ can be computed recursively from

$$
\mathcal{G}_{i}=\left(D_{i}-V_{i-1}^{T} \mathcal{G}_{i-1} V_{i-1}\right)^{-1} \quad \text { with } \mathcal{G}_{1}=D_{1}^{-1} .
$$

Finally noting that $\left(\mathbf{x}_{N}^{(n)}\right)_{l}=\langle l l|G(E)| n n\rangle$, we can now compute the localization length $\xi_{2}$ from Eq. (4). This reduces the calculation to manipulations of matrices of sizes from $1 \times 1$ to $N \times N$. It is worthwhile to point out that at the final stage of the iteration, the calculation is formally reduced to an effective $1 \mathrm{~d}$ model for a single particle. It is straightforward to generalize the algorithm to compute the other localization lengths defined in this paper. E.g., $\xi_{r}$ is calculated by decomposing Hilbert space according to the dotted lines of Fig. 1(a).

For $\xi_{2}$, we set $n=1$ in the above algorithm and obtain $t_{l, 1}=\ll \ln |\langle l l|G| 11\rangle| \gg$ with $1 \leq l \leq N$ for each parameter set $(W, U)$. We find that $t_{l, 1}$ depends linearly on $l$, implying an exponential decay of the Green function. To eliminate finite size effects near $l=1$ and $l=N$, we fit $t_{l, 1}$ in the range $N / 5 \leq l \leq 4 N / 5$ to

$$
t_{l, 1}=-\frac{l}{\xi_{2}}+c
$$

with $c$ a constant. We find that for chains $N \geq 200$, our results for $\xi_{2}$ are essentially independent of system size $N$, suggesting that finite-size effects on $\xi_{2}$ are rather weak. Similar procedures are performed for the other localization lengths.

Our main results are presented in Fig. 2. All data have been obtained for system size $N \geq 200$ and for the center of the band $E=0$. In view of the special nature of the doubly-occupied sites due to the on-site interaction, it is natural to ask whether the definition for the twoparticle localization length $\xi_{2}$ correctly captures the CM motion. To answer this question, we plot $\xi_{2, a}$ for interaction strength $U=1.0$ as function of $a$ in Fig. 2(a). We find that $\xi_{2, a}$ remains unchanged up to rather large $a$, implying that $\xi_{2}$ is indeed a good description of the CM motion. In fact, $\xi_{2, a}$ remains independent of $a$ even for $a>\xi_{1}$. Hence, as opposed to the previous beliefs [2,5] 
the interaction affects the two-particle motion even if the particle distance exceeds $\xi_{1}$. This can be understood in terms of single-particle propagation in the $2 \mathrm{~d}$ lattice of Fig. 1. $\xi_{2, a}$ is associated with the transition probability along the dashed line a distance $\sim a$ from the diagonal. We recall that when $\langle m, m-a|G| n, n-a\rangle$ is expanded in powers of the hopping matrix element $t$, it is given by a sum over all possible paths from $|n, n-a\rangle$ to $|m, m-a\rangle$. If the distance between these two sites, $\sim|m-n|$, is much smaller than $a$, the effect of the interaction would be negligible. However, $\xi_{2, a}$ is defined by the limiting behavior of $|m-n| \rightarrow \infty$ with $a$ finite, cf., Eq. (5). In this case, the contributions of paths which are sensitive to the interaction $U$ are no longer negligible and $\xi_{2, a}$ remains influenced by the interaction even though $a>\xi_{1}$.

In Fig. 2(b), we show our results for $\xi_{r}$ (symbols) which describes the decay of the Green function with relative distance. For comparison, we also plot $\xi_{2}$ (lines). At $U=0$, the two lengths are equal within the numerical accuracy, i.e., $\xi_{2} \simeq \xi_{r}$. As $U$ increases, $\xi_{r}$ remains nearly constant while $\xi_{2}$ shows a pronounced enhancement in qualitative agreement with Shepelyansky's prediction [2].

In Fig. 2(c) we plot $\xi_{f}$ (symbols) and $\xi_{2}$ (lines), where the former describes the range over which one particle moves with the other one fixed. At $U=0$, we find that $\xi_{f}$ approximately equals to $2 \xi_{2}$. As already seen in Fig. 2 (b), $\xi_{2}$ shows a strong increase with $U$. By comparison, $\xi_{f}$ shows a much weaker increase. Hence, there exists a $U_{c}(W)$ beyond which $\xi_{2}$ exceeds $\xi_{f}$.

At $U=0$, the two particles move independently and the propagation of a given particle is not affected by whether the other is moving in the reverse $\left(\xi_{r}\right)$ or in the same direction $\left(\xi_{2}\right)$, implying $\xi_{r}=\xi_{2}$. Moreover, since $\xi_{2}$ measures two-particle propagation, while $\xi_{f}$ the singleparticle motion, one expects that the transition probability for $\xi_{2}$ is given by the square of that for $\xi_{f}$, so that $\xi_{2}=\xi_{f} / 2$. We note in passing that $\xi_{2}(U=0) \neq \xi_{1} / 2$, as was pointed out in ref. [6]. Since both $\xi_{r}$ and $\xi_{f}$ are determined by the limiting behavior for diverging distance between the particles, one does not expect them to be strongly influenced by the interaction. This explains the rather flat dependences of both lengths on $U$.

With the additional information from $\xi_{r}$ and $\xi_{f}$, we can now construct a wavefunction picture in the $2 \mathrm{~d}$ lattice representation of the problem (Fig. 1). At $U=0$, this implies that the wavefunction profile is described by a square as shown by a thick solid line in Fig. 1(a). As $U$ increases, the length of the edge associated with $\xi_{2}$ increases while that associated with $\xi_{r}$ remains essentially constant. For $U>U_{c}$, the wavefunction profile becomes highly anisotropic and we find that it can be well described by an ellipse as shown by the thick solid line in Fig. 1(b). The elliptical shape predicts the relation

$$
\xi_{f}=\frac{2 \xi_{2} \xi_{r}}{\sqrt{\xi_{2}^{2}+\xi_{r}^{2}}}
$$

We have checked that our data are in good agreement with this expression for $U>U_{c}$. This clearly shows that the enhancement effect is associated predominantly with the direction of $\xi_{2}$, i.e., the $\mathrm{CM}$ motion of the two particles.

These results allow us to resolve some of the above mentioned discrepancies between previous numerical studies. We start by noting that the TMM measures the largest length scale from the $N^{2}$ Green-function entries $\langle 1 n|G| N m\rangle$ with $1 \leq m, n \leq N$ [10]. According to our results, there are two competing lengths $\xi_{2}$ and $\xi_{f}$. For $U<U_{c}$, we find that the largest length is $\xi_{f}$ while for $U>U_{c}$, it is $\xi_{2}$. Therefore, the TMM actually measures $\xi_{f}$ for $U<U_{c}$ and $\xi_{2}$ only for $U>U_{c}$. We first compare our results to those of Frahm et al. [4]. We find that their results for the localization length are two to three times larger than our result for $\max \left\{\xi_{f}, \xi_{2}\right\}$ at given values of $W$ and $U$. We attribute this to large finite-size effects in the TMM, as suggested in ref. [7]. On the other hand, in ref. [7], any enhancement effect was attributed to finite-size effects and it was suggested that the TMM produces the single-particle localization length for a sufficiently large system size. This is clearly inconsistent with the results of the present paper. We suggest the following explanation for the numerical results of ref. [7]. The argument of ref. [7] is based on TMM data for $(W, U)=(3.0,1.0)$. For these parameters, we find that the largest length is $\xi_{f}=13.2 \pm 0.3$, which is close to $\xi_{1} \simeq$ 11.7. Hence, we expect that the data in ref. [7] in fact extrapolate to $\xi_{f}$ which is indistinguishable from $\xi_{1}$ within the numerical accuracy of ref. [7]. Therefore, we contend that the principal argument of ref. [7] is a misinterpretation of data for a special parameter set and expect that the TMM exhibits the enhancement effect clearly once $\xi_{2} \gg \xi_{f}$. Finally, we find that the $\xi_{2}$ 's in ref. [5] are somewhat larger than those in this paper, which we attribute to the approximate treatment of the Green function in ref. [5].

We thank H.A. Weidenmüller for useful discussions.

[1] S.V. Kravchenko, D. Simonian, M. P. Sarachik, W. Mason and J. E. Furneaux, Phys. Rev. Lett. 77, 4938 (1996).

[2] D. L. Shepelyansky, Phys. Rev. Lett. 73, 2607 (1994); see also O. N. Dorokhov, Zh. Eksp. Teor. Fiz. 98, 646 (1990) [Sov. Phys. JETP 71, 360 (1990)].

[3] Y. Imry, Europhys. Lett. 30, 405 (1995).

[4] K. Frahm, A. Müller-Groeling, J.-L. Pichard and D. Weinmann, Europhys. Lett. 31, 169 (1995).

[5] F. von Oppen, T. Wettig and J. Müller, Phys. Rev. Lett. 76, 491 (1996).

[6] P. H. Song and D. Kim, Phys. Rev. B 56, 12217 (1997).

[7] R. A. Römer and M. Schreiber, Phys. Rev. Lett. 78, 515 (1997).

[8] F. von Oppen and T. Wettig, Europhys. Lett. 32, 741 
(1995).

[9] P. Jacquod and D.L. Shepelyansky, Phys. Rev. Lett. 78, 4986 (1997).

[10] K. Frahm, A. Müller-Groeling, J.-L. Pichard and D. Weinmann, Phys. Rev. Lett. 78, 4889 (1997); R. A. Römer and M. Schreiber, Phys. Rev. Lett. 78, 4890 (1997).

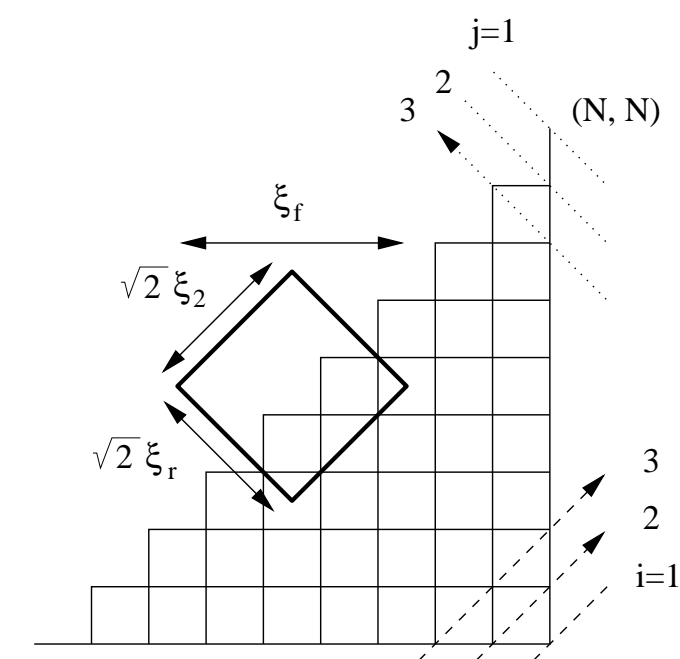

$(1,1)$

$(\mathrm{N}, 1)$

(a) $\mathrm{U}=0$

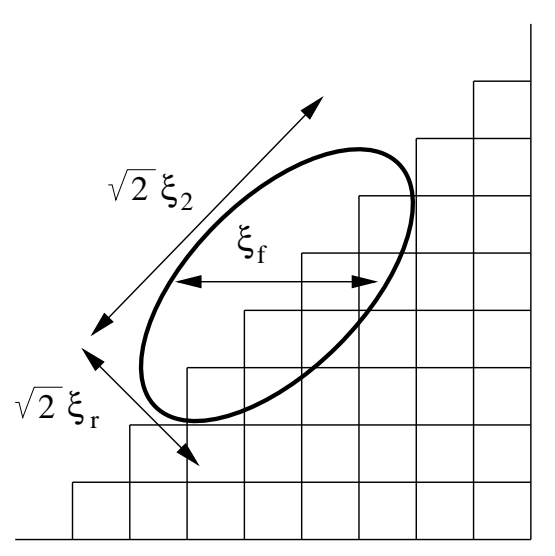

(b) $\mathrm{U}>\mathrm{U}_{\mathrm{c}}$

FIG. 1. Sketch of the two dimensional lattice (thin solid lines) and the wavefunction profile (thick solid lines). The dashed lines (dotted lines) represent the index scheme for the calculation of $\xi_{2, a}\left(\xi_{r}\right)$. Lengths are measured in terms of the lattice constant $d$. The factor $\sqrt{2}$ arises because $\xi_{r}$ and $\xi_{2}$ are defined in units of the diagonal length of the smallest square of the lattice $(\sqrt{2} d)$ while $\xi_{f}$ is defined in units of its edge length $(d)$.
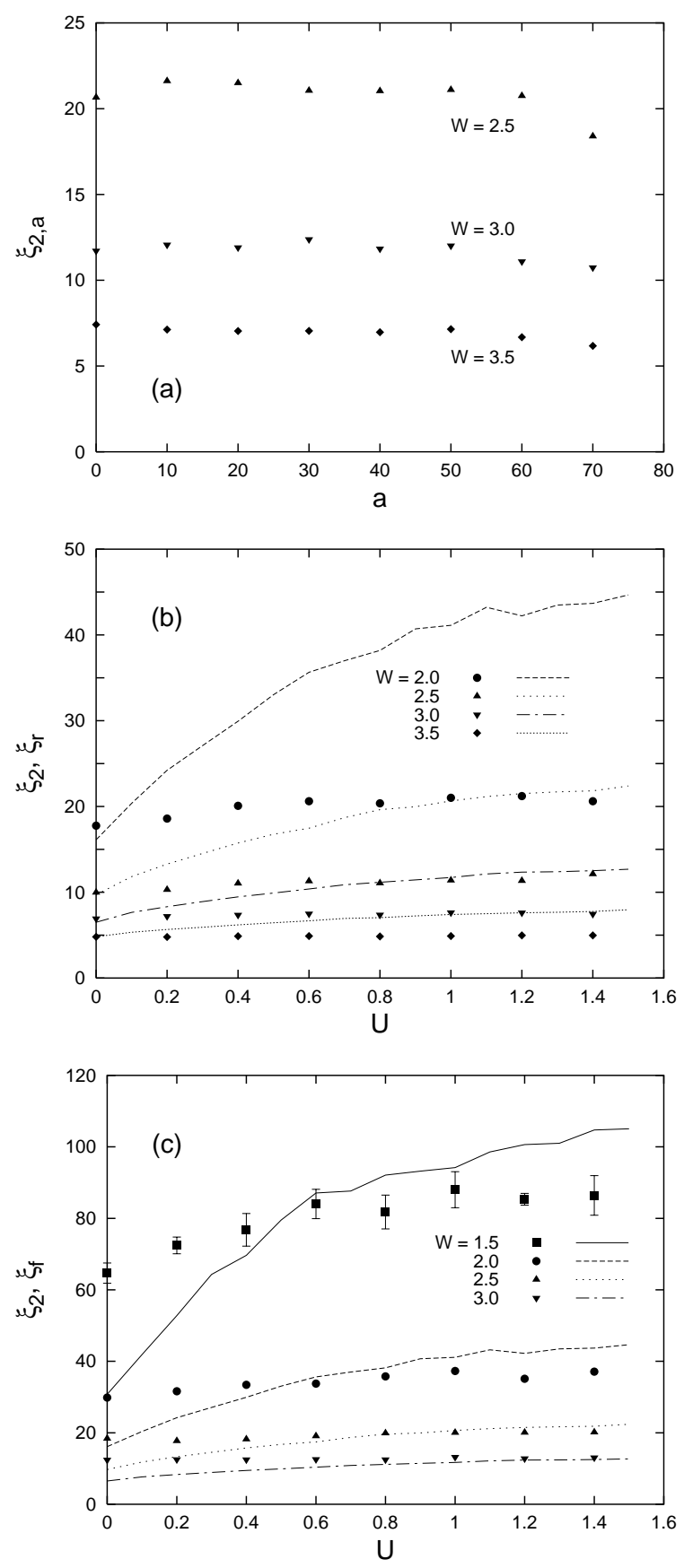

FIG. 2. (a) $\xi_{2, a}$ as function of $a$. (b) $\xi_{2}$ (lines) and $\xi_{r}$ (symbols) as function of $U$. (c) $\xi_{2}$ (lines) and $\xi_{f}$ (symbols) as function of $U$. All data have been obtained for system size $200 \leq N \leq 300$ and for $E=0$. 